\title{
LIST OF POSTER PAPERS
}

\section{A. Star Forming Regions}

A-1. Evidence for near infrared proplyd-like objects in the region around IRAS 20126+4104?, Ayala et al., p. 5

A-2. Star formation in the globules of the Trifid Nebula, Cernicharo et al., p. 8

A-3. Observations of $\mathrm{HCO}^{+}$and $\mathrm{H}^{13} \mathrm{CO}^{+}$toward the molecular cloud around HH 1-2 VLA 1, Choi \& Zhou, p. 11

A-4. Deep near-infrared and optical imaging photometry of the Serpens cloud core, Giovannetti \& Caux, p. 14

A-5. Triggered star formation in the HII region IC1848, Lefloch, Lazareff \& Castets, p. 15

A-6. The kinetic temperature structure within NGC2024, Mangum, Wootten \& Barsony, p. 18

A-7. Near infrared $\mathrm{H}_{2}$ imaging of YSOs in Vela molecular clouds, Massi, Lorenzetti \& Vitali, p. 21

A-8. CS and $\mathrm{NH}_{3}$ observations of star forming regions with $\mathrm{HH}$ objects and molecular outflows, Morata, Estalella \& López, p. 24

A-9. Small scale dust continuum structure in the $\rho$ Ophiuchi cloud cores, Motte, André \& Neri, p. 27

A-10. Near-infrared images of NGC 7129, Palacios, Eiroa $\mathcal{E}$ Miranda, p. 30

A-11. The IRAM Key-project: small-scale structure of pre-star forming regions, Panis et al., p. 33

A-12. Star formation in Cepheus IV, Plazy $\&$ Ménard, p. 36

A-13. Shocked molecular hydrogen in NGC 7129, Schultz et al., p. 39

A-14. Non-stable objects in the region of NGC 7129, Semkov, p. 42

A-15. Interferometric observations of a cometary bright-rimmed cloud in IC1396, Sugitani et al., p. 45

A-16. Infrared observations of S235 A-B complex and S233 A star forming region, Wang, Testi \& Felli, p. 48 


\section{B. Herbig-Haro objects, jets and outflows}

B-1. Herbig-Haro objects discovered at Mount Laguna Observatory, Alten et al., p. 51

B-2. The relationship between high-density gas, optical and molecular outflows, Anglada, Sepúlveda \& Gómez, p. 54

B-3. The L43 outflow: a wind-driven shell, Bence, Padman \& Isaak, p. 57

B-4. Radio continuum studies of a sample of Herbig-Haro objects in Orion, Bloemhof, p. 60

B-5. An asymmetric radio continuum HH-jet from VLA1623, Bontemps \& André, p. 63

B-6. ISO-LWS observations of IRAS16293-2422, Ceccarelli et al., p. 66

B-7. Stability and collimation of Herbig-Haro jets, Cerqueira \& $d e$ Gouveia Dal Pino, p. 70

B-8. The disk-outflow system around IRAS 20126+4104, Cesaroni et al., p. 73

B-9. $\quad \mathrm{SiO}$ in protostellar outflows, Chandler $\&$ Richer, p. 76

B-10. A mechanism for the optical emission of stellar jets, Chiuderi, Bacciotti \& Pouquet, p. 79

B-11. A Spectroscopically Observed Jet from LkH $\alpha$ 233, Corcoran $\mathcal{G}$ Ray, p. 82

B-12. Herbig-Haro objects in Sa 136, Corporon \& Reipurth, p. 85

B-13. Molecular abundance changes in the vicinity of Herbig-Haro shocks - the case of HH2, Dent, p. 88

B-14. New Herbig-Haro objects, Devine, Reipurth \& Bally, p. 91

B-15. Kinematics of the giant HH34 complex, Devine, p. 95

B-16. Numerical simulations of the Kelvin-Helmholtz instability in nonadiabatic jets, Downes, Ray and Drury, p. 98

B-17. The L1448 SiO jet, Dutrey, Guilloteau \& Bachiller, p. 101

B-18. $\mathrm{H}_{2}$ jet-like emission in the Serpens cloud core, Eiroa et al., p. 103

B-19. Near-infrared spectra of the $\mathrm{H}_{2}$ emission-line fingers in the OMC1 molecular outflow, Everett \& Pogge, p. 106

B-20. Fluorescent molecular hydrogen emission in $\mathrm{HH}$ type outflows, Fernandes, p. 109

B-21. Braking of a protostar driving magnetic x-winds, Ferreira, Pelletier \& Appl, p. 112

B-22. Numerical simulations of magnetized YSO jets: establishing a baseline, Frank et al., p. 115

B-23. The physical structure of HH29, Fridlund et al., p. 117

B-24. Submillimetre CO mapping of the outflows in HH25/26, Gibb \& Davis, p. 120 
B-25. High-velocity $\mathrm{HCO}^{+}$emission in the NGC 2071 molecular outflow, Girart et al., p. 123

B-26. The internal structure of the L 1157 molecular outflow, Gueth, Guilloteau \& Bachiller, p. 126

B-27. The origin of the fan of "bullets" in the OMC-1, Gvaramadze, p. 129

B-28. The effect of magnetic fields on jet stability and mass entrainment, Hardee, Stone \& Rosen, p. 132

B-29. A new molecular hydrogen outflow in Serpens, Herbst, Beckwith \& Robberto, p. 135

B-30. Molecular outflows and 1000 AU structure of low mass YSO envelopes, Hogerheijde et al., p. 138

B-31. Jets in the star forming region Cepheus A, Hughes, p. 141

B-32. Interferometric observations of the Class 0 object IRAS 23012 +6126 and its bipolar outflow(s), Ladd \& Howe, p. 144

B-33. Morphology and kinematics of the DG Tau jet, Lavalley, Dougados \& Cabrit, p. 147

B-34. Numerical simulations of stellar jets: emission nodes and internal shocks, Leeuwin et al., p. 150

B-35. Outflows of magnetized rotators: structure and collimation, Lery et al., p. 152

B-36. Time dependent, multidimensional models of C-shocks, Mac Low \& Smith, p. 155

B-37. The R Mon + NGC2261 jet revisited, Magakian \& Movsessian, p. 158

B-38. Monitoring the high proper motion thermal radio jet in $\mathrm{HH} 80-81$, Martí, Rodríguez \& Reipurth, p. 160

B-39. Kinematics of the molecular sheath of the HH 111 optical jet, Nagar, Vogel \& Stone, p. 163

B-40. SEST observations of the HH 135/136 complex, Ogura et al., p. 166

B-41. The interaction between Herbig-Haro jets and molecular gas in L1617, Olberg, p. 169

B-42. Infrared imaging and spectroscopy of the RNO 43 jet, Richer, Bence \& Wright, p. 172

B-43. A new Herbig-Haro object and jet from a massive B star, Shepherd \& Watson, p. 175

B-44. Ortho and para molecular hydrogen in outflows, Smith, Davis $\mathcal{G}$ Lioure, p. 178

B-45. Numerical simulations of protostellar jets, Suttner et al., p. 181

B-46. New structures in the HH24 and HH135 complexes, Tabone $\&$ Zealey, p. 184 
B-47. 2D-simulations of magnetized jets without radiative losses, Thiele \& Camenzind, p. 187

B-48. Bow shocks in non-uniform media, Wilkin, p. 190

B-49. The synchrotron jet from the $\mathrm{H}_{2} \mathrm{O}$ maser source in $\mathrm{W} 3(\mathrm{OH})$, Wilner et al., p. 193

B-50. A peculiar jet-like molecular outflow toward L1157, Zhang et al., p. 195

B-51. HH212: a prototype molecular hydrogen jet from a deeply embedded protostar, Zinnecker, McCaughrean \& Rayner, p. 198

\section{Circumstellar environments, infall and accretion}

C-1. The contribution of circumbinary envelopes to polarisation and intensity modulations, Berger \& Ménard, p. 201

C-2. Investigation of outflowing streams in PMS stars envelopes by means of spectroscopic and polarimetric methods, Beskrovnaya, p. 204

C-3. A domain decomposition computation of radiative transfer in protostellar envelopes, Chick \& Cassen, p. 207

C-4. Accretion disks irradiated by their central star. Application to T Tauri Stars, D'Alessio et al., p. 210

C-5. Gravitational collapse of a rotating cloud to form star and disk system, Hanawa, Matsumoto \& Nakamura, p. 212

C-6. Molecular and atomic shocks in the near environment of T Tauri, Herbst, Robberto \& Beckwith, p. 215

C-7. Imaging of the unusual T Tauri star V1331 Cyg with surrounding nebula, Hojaev \& Zheleznyak, p. 218

C-8. Detailed self-consistent model of the dusty disk around HL Tau, Men'shchikov, Henning \& Fischer, p. 221

C-9. The structure of disks and envelopes around young stars: new results from ISO, Meyer, Beckwith \& Natta, p. 224

C-10. Near-infrared and millimeter observations of the S255 region, $M i$ ralles et al., p. $\mathbf{2 2 7}$

C-11. Circumstellar environment of close PMS binary systems, Monin, Geoffray \& Ménard, p. 230

C-12. Dynamical collapse of magnetized molecular clouds: formation of self-similarly collapsing disks, Nakamura, Hanawa $\&$ Matsumoto, p. 232

C-13. Non-axisymmetric evolution of dynamically contracting disks and formation of binary stars, Nakamura \& Hanawa, p. 235

C-14. IRAS sources associated with small nebulae in dark clouds, Persi et al., p. 238 
C-15. Three dimensional simulations of protostellar disks: two extremes in cooling, Pickett, Cassen \& Durisen, p. 241

C-16. Spatial and kinematical stratification in the gaseous envelopes of PMS objects, Pogodin \& Vieira, p. 244

C-17. Formation of a dynamically contracting protostellar disk, Saigo \& Hanawa, p. 247

C-18. Fragmentation, contraction, and accretion in magnetized clouds, Tomisaka, p. 250

C-19. Simulations of a supersonic accretion onto magnetized disks: properties of developing outflows, Toropin, Saveljev \& Chechetkin, p. 254

C-20. ISOPHOT observations of a pre-stellar core, Ward-Thompson et al., p. 257

C-21. The spectral signature of accretion in low-mass protostars: observations and NLTE modelling, Wiesemeyer, Güsten $\mathcal{G}$ Wright, p. 260

\section{Central stellar objects and their winds}

D-1. Modulated temporal variability in DF Tauri: a bipolar Br $\gamma$ emission structure, Chelli et al., p. 263

D-2. The powering sources of the quadrupolar molecular outflow in L723, Estalella et al., p. 266

D-3. Looking for the spectral fingerprints of some mechanisms responsible of the T Tauri stars variability, Fernández et al., p. 269

D-4. Winds and accretion in T Tauri stars: Paschen Beta line profiles, Folha, Emerson \& Calvet, p. 272

D-5. Line profile variations in the classical T Tauri star DR Tau, Gameiro and Lago, p. 275

D-6. UV variability in BP Tau, Gómez de Castro \& Franqueira, p. 278

D-7. On the stability of initial epochs and photometric periods of weakline T Tauri stars, Grankin, p. 281

D-8. The spectral energy distribution of HH 100 IRS, Gredel, Siebenmorgen \& Starck, p. 284

D-9. Photometric observations of YY Orionis, Harder et al., p. 288

D-10. Far-infrared observations of SSV 13 in NGC 1333, Harvey, p. 291

D-11. Multicolour polarimetric observations of T Tauri stars, Heines, Henning \& Szeifert, p. 294

D-12. Data bank on photoelectric UBVR observations of the FUors V1057 Cyg, V1515 Cyg, and FU Ori (1981-1995), Ibrahimov, p. 297

D-13. Doppler imaging of SU Aurigae, Mennessier, p. 300 
D-14. Alfvén wave-heated winds of T Tauri Stars, Paatz $\&$ Appl, p. 303

D-15. High resolution studies of $\mathrm{H} \alpha$ emission in young stars, Pedrosa, p. 306

D-16. On the variability of the FUor BBW 76, Reipurth, p. 309

D-17. Protostellar cosmic rays and extinct radioactivities, Shang et al., p. 312

D-18. Rapid variations of Classical T Tauri emission features. The case of DR Tau, Smith et al., p. 315

D-19. Diamagnetic blob interaction model of $\mathrm{T}$ Tauri variability, Ultchin, Regev \& Bertout, p. 318

\section{E. Additional posters}

Available only in the Web version

E-1. Two interesting examples of jets from YSOs: Haro 6-10 and CoKu Tau/1, Movsessian \& Magakian, p. W01

E-1. Far infrared spectroscopy of the B335 region, Nisini et al., p. W03

E-1. HH ejecta and the heating of the Orion Ridge, Wiseman, Putman \& $\mathrm{Ho}$, p. W06

E-1. The Herbig-Haro knots of Orion, Burton, p. W09

\section{How to get the poster papers}

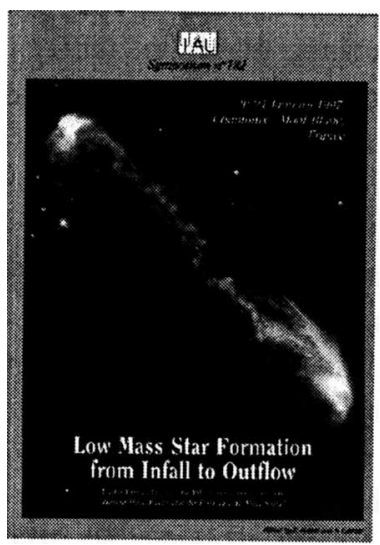

The poster book has been distributed to all the participants at the conference and to most of the main astronomical libraries. No further copies are available. However, the poster book is available on the Web at the following address:

http://www-laog.obs.ujf-grenoble.fr/meetings/iau182 


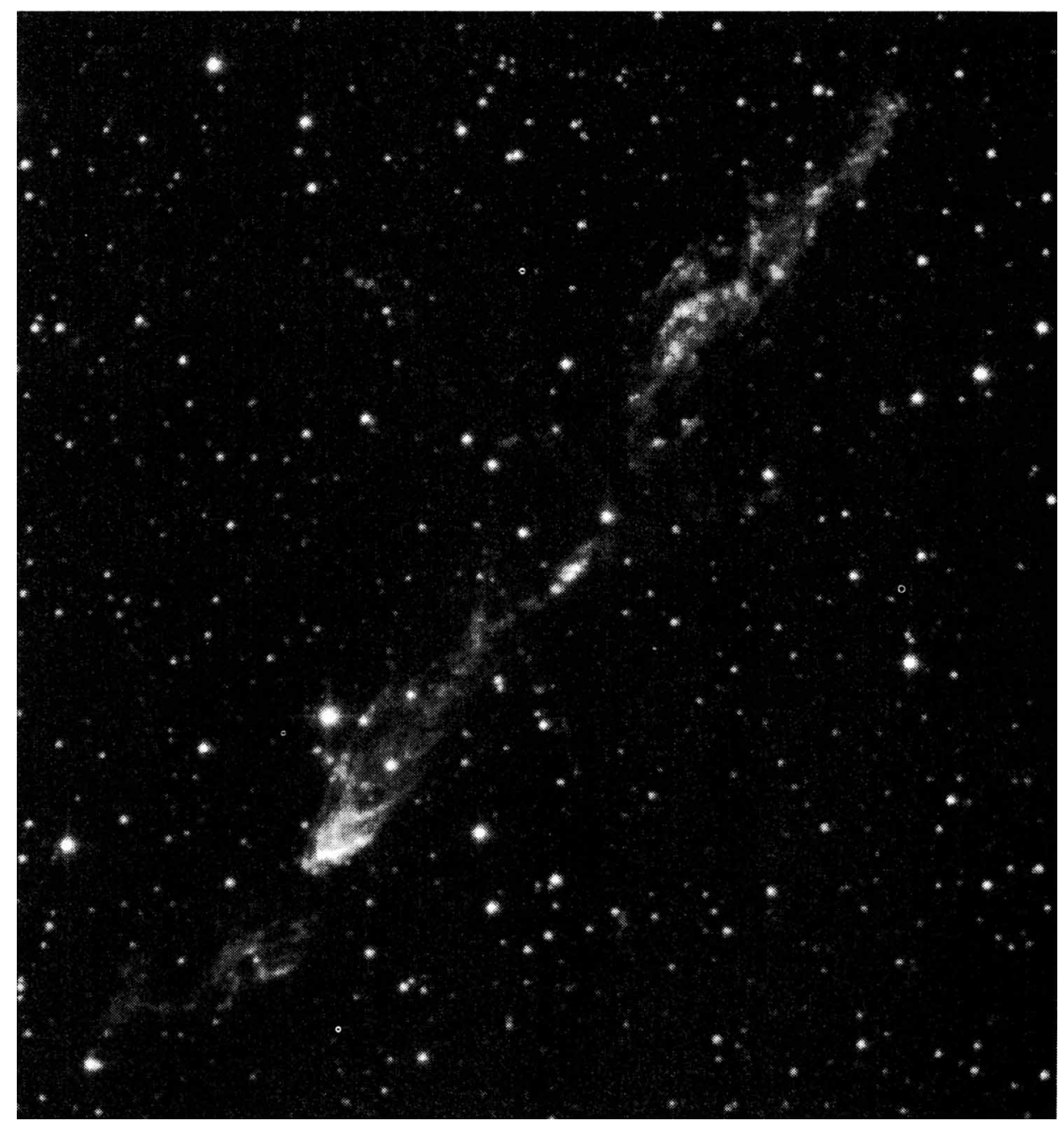

I am an unquenchable fire, the centre of all energy, the stout heroic heart; I am truth and light, I hold power and glory in my sway; My presence disperses dark clouds, I have been chosen to tame the fates; I am the Dragon

\section{- T. Low, The Handbook of Chinese Horoscopes}

This molecular hydrogen image ( $\mathrm{v}=1-0 \mathrm{~S}(1)$ line at $2.122 \mu \mathrm{m})$ shows HH 288 in Cassiopeia, dubbed the Dragon Jet after its resemblance to the mythical fire-breather. The jet emanates from a deeply embedded young source and drives an associated molecular outflow; at the $2 \mathrm{kpc}$ kinematic distance of the ambient cloud, the jet is over 3 parsec long.

Data taken by Mark McCaughrean using Omega-Prime on the Calar Alto 3.5-m; 0.8 arcsec seeing; 33 minutes integration time; $4.3 \times 4.6$ arcmin field; continuum not subtracted. 